\title{
Bulk-Driven Nonequilibrium Phase Transitions in a Mesoscopic Ring
}

\author{
Hauke Hinsch and Erwin Frey \\ Arnold Sommerfeld Center for Theoretical Physics and Center of NanoScience, Department of Physics, \\ Ludwig-Maximilians-Universität München, Theresienstrasse 37, D-80333 München, Germany \\ (Received 26 March 2006; published 30 August 2006)
}

\begin{abstract}
We study a periodic one-dimensional exclusion process composed of a driven and a diffusive part. In a mesoscopic limit where both dynamics compete we identify bulk-driven phase transitions. We employ mean-field theory complemented by Monte Carlo simulations to characterize the emerging nonequilibrium steady states. Monte Carlo simulations reveal interesting correlation effects that we explain phenomenologically.
\end{abstract}

DOI: 10.1103/PhysRevLett.97.095701

One-dimensional (1D) driven diffusive systems [1] have not only served as fruitful testing grounds for fundamental questions in nonequilibrium physics [2] but have also been the focus of recent interest in applications relevant to biological problems [3,4]. Restricted 1D motion may either result from geometric confinement as in a nuclear pore complex of cells [5] or artificial crystalline zeolitical structures [6], or arise because molecular engines move along one-dimensional tracks as, for example, in intracellular transport [7] and protein synthesis [8,9].

In these systems interesting collective effects emerge since mutual passage of particles is excluded. The nature of these effects depends on whether the system is purely diffusive (passive) or driven due to the presence of an external field or an internal driving mechanism inherent to the particles (active), e.g., motor activity in intracellular transport. In addition, one has to distinguish between open and closed boundary conditions. Independent of their boundary conditions, both active and passive systems show interesting dynamic anomalies [10-12]. In contrast, only systems with open boundaries are known to exhibit nontrivial nonequilibrium steady states [13].

Inspired by traffic of molecular motors in closed compartments [3] and colloidal motion in optical traps [12], we present a model that combines the symmetric and the totally asymmetric simple exclusion process (SEP and TASEP, respectively). It is intended to investigate the competition between driven and diffusive motion in 1D systems. While the breaking of translational invariance is known to be necessary for nontrivial steady states to evolve, we show that additionally time scale separation between the two processes requires a mesoscopic scaling to guarantee a finite current and a physical behavior in the continuum limit.

SEP and TASEP serve as the two paradigms for passive and active transport in one dimension. In these lattice gas models particles occupy the sites of a 1D lattice subject to the simple exclusion rule that each site may be occupied by at most one particle. In the SEP particles jump independently and randomly at rate $D$ to vacant neighboring sites with equal probabilities to the left and right, while hopping
PACS numbers: 64.60.Ht, 05.40.-a, 05.60.-k, 05.70.Ln

at a rate $R$ is strictly unidirectional for the TASEP. In the following we will measure time in units of $1 / R$; i.e., we set $R=1$. For a closed ring geometry both processes are characterized by a steady state with a uniform density profile since translational invariance gives equal weight to all permissible configurations. For open boundary conditions, the steady state of the SEP always shows a linear density profile [14], whose slope depends on the boundary condition's difference. In stark contrast, TASEP exhibits several distinct nonequilibrium phases [13] as a function of the magnitude of the entrance and exit rate, $\alpha$ and $\beta$, at the left and right boundary, respectively. Two different phases can be characterized by their global particle density (low and high) and one by a maximal current. The validity of the initial mean-field analysis of the phase diagram was later supported by several exact solutions [15].

In this Letter we aim at identifying the nature of the nonequilibrium steady states of a closed ring system consisting of two equally sized compartments with $i=$ $1, \ldots, N$ lattice sites, whose dynamics is governed by a symmetric and a totally asymmetric exclusion process, respectively; see Fig. 1. To distinguish between the active and passive compartments of the ring, quantities for the passive part like the location of the lattice sites, $\tilde{x}_{i}$, or the occupation numbers $\tilde{n}_{i} \in\{0,1\}$ are indicated by a tilde. Particle exchange between both sublattices is exclusively allowed at their junctions with dynamic rules defined by the originating site. Thus a vacancy on the right-hand side

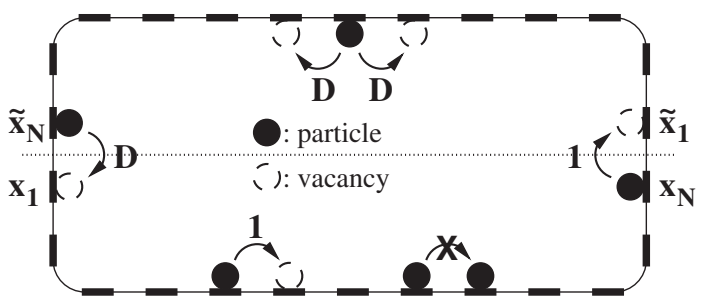

FIG. 1. Schematic model of the ring system. The dynamics of the upper (passive) lane with lattice sites $\tilde{x}_{i}$ is governed by a SEP with rate $D$, and the dynamics of the lower (active) lane with lattice sites $x_{i}$ by a TASEP with unity jump rate. 
(r.h.s.) $\tilde{x}_{1}$ of the passive part can be filled with a particle from site $x_{N}$ of the active section with rate unity, while the corresponding event at the left junction will occur with rate $D$. Since the system is closed, the particle number $N_{p}=$ $\sum_{i=1}^{N}\left(n_{i}+\tilde{n}_{i}\right)$ is conserved and the particle density

$$
n_{p}=\frac{N_{p}}{2 N}
$$

serves as a dimensionless control parameter confined to the interval $[0,1]$. Having chosen the inverse hopping rate $1 / R$ on the active part as our time unit, we are left with a twodimensional parameter space $\left(n_{p}, D\right)$. As these parameters are bulk quantities, resulting phase transitions will be bulkinduced in contrast to boundary-induced phase transitions in TASEP. In order to explore the phase behavior in this parameter space we will exploit the fact that for a closed system the steady state current $J\left(n_{p}, D\right)$ is spatially constant.

We analyze the ring system by mean-field (MF) theory complemented by Monte Carlo simulations (MCS). The MF analysis allows one to decouple the two parts of the ring and consider them as separate lanes with effective entrance and exit rates. Once these rates are identified we can use known results for the SEP and TASEP with open boundaries. To begin with, we introduce the following notation for the stationary densities $\rho_{i}=\left\langle n_{i}\right\rangle$ at the junction sites: $\gamma=\tilde{\rho}_{1}$ and $\delta=\tilde{\rho}_{N}$ for the passive part, and $\alpha=\rho_{1}$ and $1-\beta=\rho_{N}$ according to TASEP convention for the active part. The incoming current to site $x_{1}$ is the product of the average occupation number on the originating site, the jump rate to the destination site, and the probability that the latter is empty: $\delta D(1-\alpha)$. Because of current conservation this has to equal the current to site $x_{2}: \alpha(1-\alpha)$. Using particle-hole symmetry, one can proceed accordingly at the other junction to arrive at the following relations:

$$
\alpha=D \delta, \quad \gamma=1-\beta .
$$

To analyze the interplay of the two junctions, we will exploit the conservation of current mentioned above. On the active part, directed motion results in a current of $J=$ $\rho_{i}\left(1-\rho_{i+1}\right)$. Conservation of current can be fulfilled only for a spatially constant or piecewise constant density distribution. In the latter case, two sections of constant density $U$ and $V$ are connected by a domain wall and their densities have to fulfill the condition $\rho_{U}=1-\rho_{V}$ to conserve the current.

As particle motion is bidirectional on the passive part, the current between two sites is obtained as the balance of their bilateral particle exchange, which is proportional to their density difference. Conservation of current thus demands a linear density slope and the current takes the form $J=(\gamma-\delta) D / N$ reminiscent of Fick's law [16]. Evidently the passive part current vanishes with system size, while the active part current is constant. Because of current conservation this implies that the smaller current, and thus the diffusive process, is dominating the system in a trivial way. To broaden our analysis to a wide parameter range for arbitrary system sizes we introduce a mesoscopic scaling [4] and a new control parameter:

$$
d=\frac{D}{N}
$$

This scaling can be understood as a time scale separation and ensures competitive behavior between the system's constituents. The specific form (3) even guarantees a well-behaved approach to the continuous case $N \rightarrow \infty$. With the new control parameter the passive part current is expressed as $J=d(\gamma-\delta)$ and allows for a relation between the two junctions:

$$
\delta=\gamma-\frac{J}{d}
$$

Having derived suitable entry and exit rates for the active part, we can now apply the TASEP results. The low (high) density (LD and HD, respectively) phases are realized in the periodic system equally and are characterized by a uniform density below (above) $1 / 2$ and a boundary layer at the right (left). Special attention has to be paid to the phase boundary $\alpha=\beta$ between the LD and HD phase, where the boundaries are matched by a piecewise constant density profile with an intervening domain wall (DW). Because of the randomness of entry and exit events this DW is delocalized and subjected to a random walk that explores the complete system on long time scales. In the ring system, however, our MCS reveal a DW localization (Fig. 2). We understand this as a consequence of entry and exit rates that are not statistically independent as in TASEP but rather connected via the passive part, dependent on the amount of available particles and the diffusion rate.

To proceed with a quantitative analysis, we connect the boundary conditions of the two sublattices in a self-

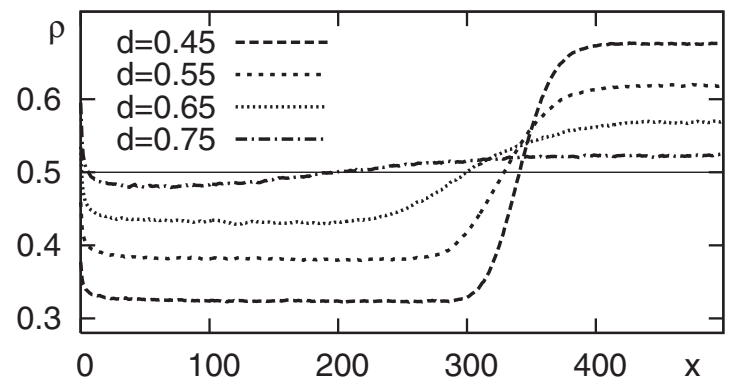

FIG. 2. Simulated density profiles of the active part in a system of $N=500$ and $n_{p}=0.38$ for different diffusion strengths $d$ (indicated in the graph). The system is in the LD-HD phase featuring a domain wall. With increasing $d$ the domain wall position (intersection with the solid line) is shifted to the left [see Eq. (5)] and DW fluctuations increase. The left boundary layers are due to current limitation caused by correlations at the passive part boundaries (see text). 
consistent manner to obtain a junction density solely dependent on the two control parameters. The precondition $\alpha=\beta$ guarantees a conserved active part current of $J=$ $\alpha(1-\alpha)$. This allows one to rewrite Eq. (4) as $\delta=(1-$ $\alpha)[1-\alpha / d]$. Since $\delta$ is of order $1 / N$ in the thermodynamic limit (TL) $N \rightarrow \infty$, we find $\alpha=d+O(1 / N)$. Having derived $\alpha$, the remaining densities $\beta, \gamma$, and $\delta$ are easily calculated and we can proceed to connect the position of the DW to the global particle density. To this end continuous density distributions are assumed, which are applicable for large systems where the lattice spacing vanishes. To begin with, a DW is assumed to be present on the active part. Hence, a Heaviside function that connects two regions of constant density at DW position $x_{w}$ is chosen as $\rho(x)=\alpha+\Theta\left(x-x_{w}\right)(1-\alpha-\beta)$. For the passive part's density distribution a linear slope $\tilde{\rho}(x)=$ $\delta+(\gamma-\delta) x$ is appropriate. Now particle conservation can be expressed as $2 n_{p}=\int_{0}^{1} d x[\tilde{\rho}(x)+\rho(x)]$. Solving this in the TL, the DW position evaluates to

$$
x_{w}=\frac{-3+3 d+4 n_{p}}{4 d-2} .
$$

The DW position is left to depend on the two control parameters: the diffusion strength (see Fig. 2 for an example) and the particle density. The resulting $\left(d, n_{p}\right)$-phase diagram exhibits several phase transitions and regimes of which three can be characterized by the domain wall position. In the case $x_{w} \leq 0$ the DW has left the active part at the left junction resulting in a HD phase with constant density $\rho(x)=1-\beta$. A constant low density corresponding to the left boundary condition $\rho(x)=\alpha$ is established for $1 \leq x_{w}$, while the DW is localized inside the system for $0<x_{w}<1$ connecting the two boundaries by a phase of coexistence (LD-HD). Its phase boundaries can be obtained from Eq. (5) as $d=\left(3-4 n_{p}\right) / 3$ for $x_{w}=$ 0 and $d=4 n_{p}-1$ for $x_{w}=1$. The phase boundaries intersect at a critical point at $\left(n_{p}=3 / 8, d=1 / 2\right)$ where the DW height vanishes (Fig. 3). The extremal points at

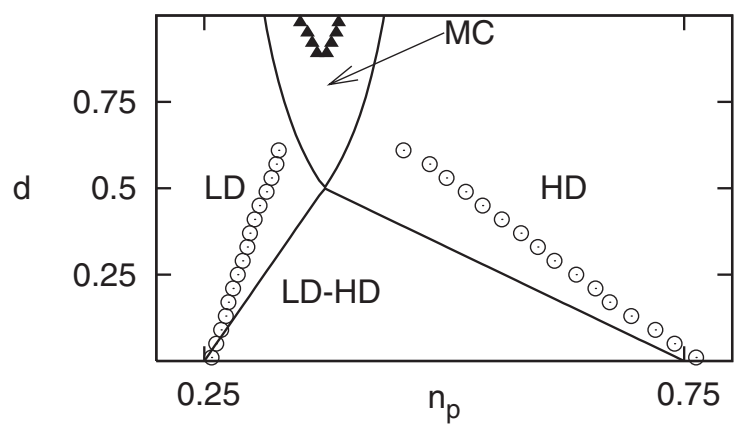

FIG. 3. Phase diagram obtained by MF (solid lines) exhibits four phases. Simulations [MC (triangles) and LD-HD (circles) phase boundaries, $N=200$ ] reveal a failure of the MF analysis: correlations at the passive part boundaries cause a diminished current that shifts the MC phase. $d=0$ of the LD-HD phase can be readily explained by the particle fraction of a quarter that has to be bound on the passive part for vanishing diffusion (signifies $\alpha=\beta=0$ ). Hence, at $n_{p}=1 / 4$ there are no particles available to the active part and the system crosses over into the LD phase. Analogous, the HD phase is entered for values of $n_{p}>$ $3 / 4$. The existence of the LD-HD phase is a distinctive difference to TASEP [18]. It replaces one discontinuous phase transition with two continuous transitions that meet at a multicritical point.

Similar to TASEP, the ring system also features a maximal-current (MC) phase. In this regime the active part current imposes its maximum $J_{\mathrm{MC}}=1 / 4$ on both lanes. MF approximations allow one again to derive the extent of the MC phase in dependence of the two control parameters $d$ and $n_{p}$. To this end we have to assume a different density distribution than above for the active part. The corresponding distribution in TASEP is established for boundary conditions $\alpha, \beta>1 / 2$ and has to be constant at $\rho=1 / 2$ with the exception of possible boundary layers that vanish in the TL. Hence, $N / 2$ particles have to be present on the active part. We can then deduce from particle conservation: $2 n_{p}-1 / 2=\delta+(\gamma-\delta) / 2$, where the r.h.s. is just the integral over the passive part's linear density distribution. Using the equality of the passive part and the active part current allows one to solve for $\delta=$ $2\left(n_{p}-1 / 4\right)-1 /(8 d)$. The active part constraints can be rewritten as constraints on the passive part by use of Eqs. (2) and (4) to $\delta>1 /(2 d N)$ and $\delta<1 / 2-1 /(4 d)$. The last three equations constitute planes in the $\left(\delta, d, n_{p}\right)$ space. Computing now the intersection of the first plane with the two inequalities, one can finally deduce the phase boundaries of the MC phase in $\left(d, n_{p}\right)$-phase space in the TL as $d=1 /\left(16 n_{p}-4\right)$ and $d=1 /\left(8-16 n_{p}\right)$, where the latter is the boundary with the HD phase. Notice that the former phase boundary could already be derived by considering only terms of order 1 in the TL (i.e., with $\delta=$ $0)$, while the HD boundary is obtained only if $\delta=O(1 / N)$ is considered. The MC phase originates at the critical point in phase space and asymptotes for $d \rightarrow \infty$ at values of $1 / 2$ and $1 / 4$ (see Fig. 3 ) as can easily be explained. At particle densities below $n_{p}=1 / 4$ there are not enough particles available to establish a constant density of $\rho=1 / 2$ on the active part. On the contrary, the system makes a transition to the HD phase if both active and passive part are half filled at $n_{p}=1 / 2$. At this point, $\gamma>1 / 2$ implies a violation of the MC phase requirement $\beta>1 / 2$.

We have complemented our MF analysis by extensive MCS. Their results deviate from the phase diagram derived above (see simulation data in Fig. 3). The key difference is that the multicritical point is shifted to higher values of $d$. To rationalize this we compare the density profiles from simulations and MF theory. While the density distribution on the passive part has been assumed to be linear according to SEP results, simulated data exhibit a distinct curvature in 
the profile [19]. A closer examination of the present boundary conditions reveals that these are different from open boundaries used for the derivation of the linear SEP density profile. As particles enter the passive part with a rate of unity while the internal dynamics of the diffusion are much faster, the SEP experiences a boundary that is reflective for a considerable amount of time. Like in other problems, exhibiting time scale separation, as forest-fire models [20] the stationary state results from competition between a process that strives towards equilibrium (diffusion) and another process repeatedly driving the system to nonequilibrium (particle entrance). The rare particle entrance events provide a particle excess at the right boundary. This density surplus then spreads into the bulk by diffusion before the next entrance restores the particle excess [21]. A time average then results in a strictly convex density profile on the passive part. Its curvature renders Fick's law for the passive part current inappropriate because the smallest gradient (here at the left interface) acts as a current bottleneck. The consequences for the phase diagram are obvious: since the current is effectively reduced, the MC phase is established only at higher diffusion values (Fig. 3). The current limitation caused by the bottleneck is also responsible for the boundary layers on the left-hand side. of the active part (Fig. 2). Here global conservation of current forces the density to decay quickly to a value conform with the system's minimal current. Thereby the extension of the LD-HD phase to values of $d>1 / 2$ can be explained. By introducing a correction factor for the reduced current, a refined MF theory can explain the shift of the MC phase at least qualitatively [21]. Recalculation of the LD-HD phase boundaries in this realm completely fails, also due to correlation effects on the left interface.

Our analytical and numerical studies have shown that the presented system exhibits a rich phase behavior unexpected for periodic systems. While boundary-induced phase transitions in exclusion processes occur in several geometries [4,13] and nontrivial nonequilibrium steady states are known in periodic systems if translational symmetry is broken by defects [22], comparable bulk-induced phase transitions have not been studied, to our knowledge, so far. Crucial to this behavior is the competition between the two processes, which the scaling Eq. (3) ensures for a broad parameter range. The time scale separation between the subprocesses is also responsible for the quantitative failure of MF that comes as quite a surprise, since up to now similar approximations have been known as a reliable tool to reproduce the phase diagram of lattice gas systems with an astonishing accuracy.

For the system presented, these characteristics indicate the existence of interesting correlation phenomena that call for analytical methods beyond MF. The phenomenological explanation and the modified MF theory should therefore only be considered preliminary. As MCS near the multicritical point require considerable computation resources, we cannot give any reliable predictions about the exact phase topology. The exact form of the phase diagram and the behavior near the multicritical point remain an open question to whose solution different techniques like Bethe ansatz [14] or density matrix renormalization group [23] may contribute. Furthermore, possible experimental realizations [12] could make this system an intriguing problem to study.

The authors thank P. Pierobon for helpful discussions.

[1] B. Schmittmann and R. Zia, in Phase Transitions and Critical Phenomena, edited by C. Domb and J. Lebowitz (Academic Press, London, 1995).

[2] B. Derrida, Phys. Rep. 301, 65 (1998).

[3] R. Lipowsky, S. Klumpp, and T. M. Nieuwenhuizen, Phys. Rev. Lett. 87, 108101 (2001).

[4] A. Parmeggiani, T. Franosch, and E. Frey, Phys. Rev. Lett. 90, 086601 (2003).

[5] I. Kosztin and K. Schulten, Phys. Rev. Lett. 93, 238102 (2004).

[6] J. Kärger and D. Ruthven, Diffusion in Zeolites and Other Microporous Solids (Wiley, New York, 1992).

[7] J. Howard, Mechanics of Motor Proteins and the Cytoskeleton (Sinauer Associates, Sunderland, 2001).

[8] J. MacDonald, J. Gibbs, and A. Pipkin, Biopolymers 6, 1 (1968).

[9] T. Chou, Biophys. J. 85, 755 (2003).

[10] P. M. Richards, Phys. Rev. B 16, 1393 (1977).

[11] M. Kollmann, Phys. Rev. Lett. 90, 180602 (2003).

[12] Q.-H. Wei, C. Bechinger, and P. Leiderer, Science 287, 625 (2000)

[13] J. Krug, Phys. Rev. Lett. 67, 1882 (1991).

[14] G. Schütz, in Phase Transitions and Critical Phenomena, edited by C. Domb and J. Lebowitz (Academic Press, London, 2000).

[15] B. Derrida, E. Domany, and D. Mukamel, J. Stat. Phys. 69, 667 (1992).

[16] This might appear contradictory to the known non-Fickian behavior of SEP, but is due to the gradient established by the active part. It causes a bias that invalidates the subdiffusive mean-square displacement [17].

[17] S. N. Majumdar and M. Barma, Phys. Rev. B 44, 5306 (1991).

[18] Additionally, the variance of the DW position decays as $N^{-1}$. Contrary to the $N^{-1 / 2}$ decay in TASEP, this is caused by the additional feedback via the passive part.

[19] We have conducted extensive simulations to ensure that the curvature is preserved at long times. There is no evidence that would hint at a decrease in convexity with either increasing simulation time or system size.

[20] B. Drossel and F. Schwabl, Phys. Rev. Lett. 69, 1629 (1992).

[21] H. Hinsch and E. Frey (to be published).

[22] S. A. Janowsky and J. L. Lebowitz, Phys. Rev. A 45, 618 (1992).

[23] U. Schollwöck, Rev. Mod. Phys. 77, 259 (2005). 